Agronomía Costarricense 34(1): 1-15. ISSN:0377-9424 / 2010

www.mag.go.cr/rev_agr/index.html www.cia.ucr.ac.cr

\title{
ANÁLISIS DE CRECIMIENTO Y ABSORCIÓN DE NUTRIMENTOS DE FRUTOS DE MANGO, CULTIVARES TOMMY ATKINS Y KEITH
}

\author{
Róger Fallas $^{1 *}$, Floria Bertsch ${ }^{*}$, Enaldo Miranda ${ }^{* *}$, Carlos Henríquez \\ Palabras clave: Mangifera indica, desarrollo del fruto, absorción de nutrimentos, grados día. \\ Keywords: Mangifera indica, fruit development, nutrient absorption curves, heat units.
}

Recibido: 09/11/09

Aceptado: 10/02/10

\section{RESUMEN}

Frutos de mango de cultivares Tommy Atkins y Keith fueron colectados en diferentes bloques de la Finca Manga Rica, Guanacaste, Costa Rica, a lo largo del ciclo de desarrollo durante la temporada 2008-2009. Aproximadamente cada 15 días, a los frutos colectados se les midió su ancho y largo, peso fresco y seco y se analizó la concentración de nutrimentos. Se determinó que el fruto presenta 2 fases de desarrollo, una de expansión, que abarca desde el inicio hasta los 79 días después de floración en Tommy Atkins y hasta los 93 días para Keith, y otra posterior de llenado. Se obtuvieron las curvas de absorción para macro y microelementos y se detalla información para adaptarlas a diferentes localidades. Se determinó que cada $\mathrm{kg}$ de fruta fresca de Tommy Atkins y Keith, respectivamente, consume 1,54 y $1,59 \mathrm{~g}$ de $\mathrm{K}$; 1,07 y 1,06 g de $\mathrm{N} ; 0,20$ y $0,12 \mathrm{~g}$ de P; 0,29 y $0,14 \mathrm{~g}$ de Ca; 0,19 y $0,15 \mathrm{~g}$ de $\mathrm{Mg} ; 0,07$ y $0,09 \mathrm{~g}$ de $\mathrm{S} ; 8,5$ y $2,5 \mathrm{mg}$ de Fe; 3,5 y $2,1 \mathrm{mg}$ de $\mathrm{Mn}$; 0,7 y $1,6 \mathrm{mg}$ de $\mathrm{B} ; 1,2$ y $0,97 \mathrm{mg}$ de $\mathrm{Zn}$; y 1,1 y $0,9 \mathrm{mg}$ de $\mathrm{Cu}$. Se sugieren etapas adecuadas para realizar fertilizaciones foliares y se encontró que algunos elementos no deben aplicarse simultáneamente, por posibles antagonismos. Para obtener frutos de Tommy Atkins con peso fresco entre 400 y $500 \mathrm{~g}$ se debe cosechar cuando el diámetro fluctúe entre 8,7 y $9,0 \mathrm{~cm}$, o el largo entre 10,7 y 11,2 cm. Para obtener frutos

\footnotetext{
1/ Autor para correspondencia. Correo electrónico: rafallasc@gmail.com

Centro de Investigaciones Agronómicas, Universidad de Costa Rica. San José, Costa Rica.
}

\begin{abstract}
Growth analysis and nutrient absortion curves of mango fruits, cultivars Tommy Atkins and Keith. Fruits of mango (Tommy Atkins and Keith) were collected in different blocks of the Farm Manga Rica, Guanacaste, Costa Rica, along their development cycle during the season 2008-2009. The fruits were collected approximately every 15 days, their its dimensions (width and length), weight (wet and dry) were measured, and then the concentration of nutrients was analyzed. The mango fruit has 2 phases at its development cycle, one of expansion, which starts at the beginning of the cycle and continues until 79 days after flowering in the case of cv. Tommy Atkins, and 93 days for cv. Keith; in the following phase the fruit increases in weight. Absorption curves for macro and micro nutrients were obtained, and the information to adapt the absorption curves to other localities is detailed. It was determined that each $\mathrm{kg}$ of fresh fruit of Tommy Atkins and Keith, respectively, consumed 1.54 and $1.59 \mathrm{~g} \mathrm{~K} ; 1.07$ and $1.06 \mathrm{~g}$ $\mathrm{N} ; 0.20$ and $0.12 \mathrm{~g} \mathrm{P} ; 0.29$ and $0.14 \mathrm{~g} \mathrm{Ca} ; 0.19$ and $0.15 \mathrm{~g} \mathrm{Mg} ; 0.07$ and $0.09 \mathrm{~g}$ of $\mathrm{S} ; 8.5$ and $2.5 \mathrm{mg} \mathrm{Fe} ; 3.5$ and $2.1 \mathrm{mg} \mathrm{Mn} ; 0.7$ and $1.6 \mathrm{mg}$ B; 1.2 and $0.97 \mathrm{mg} \mathrm{Zn}$; and 1.1 and $0.9 \mathrm{mg} \mathrm{Cu}$. Also; appropriate stages are suggested for foliar fertilization and it was found that some nutrients shouldn't be applied simultaneously, because of possible antagonisms. Field measurements
\end{abstract}

** Manga Rica S.A. Guanacaste, Costa Rica. 
de Keith con un peso entre 600 y 750 g, se debe colectar cuando el diámetro varíe entre 9,4 y $9,8 \mathrm{~cm}$ o el largo entre 13,5 y $14,0 \mathrm{~cm}$, según el análisis de regresión.

\section{INTRODUCCIÓN}

Es destacada la importancia que tiene el cultivo del mango en los mercados internacionales; junto con el banano, aguacate y piña se ubica dentro de los frutos tropicales más cotizados (Silva et al. 2002).

En plantaciones comerciales las mayores producciones se obtienen cuando el árbol tiene entre 8 y 15 años, cerca de los 28 años que se obtienen producciones económicamente rentables, pero menores a las obtenidas en el período anterior y después de los 28-30 años se inicia una fase de bajos rendimientos (Ríos y Corella 1999).

Algunos autores concuerdan en que existe un vacío en el manejo de la fertilización en el cultivo, aunque se tiene claro que se debería tomar en cuenta que el árbol en sus diferentes estados de desarrollo presenta diferentes requerimientos nutricionales (Avilán 1999, Silva et al. 2002), además Ponchner et al. 1993 y Silva et al. (2002) destacan que se trata de un árbol muy eficiente en la absorción de nutrimentos. Dentro de los estudios realizados algunos se han enfocado en mejorar las técnicas, épocas y métodos de fertilización y en otras latitudes se tiene información sobre la extracción de nutrimentos por parte de los frutos de algunos cultivares e inclusive sobre (equatorial diameter and length) in both cultivars would be useful to establish the time of harvest; to obtain fruits of Tommy Atkins with fresh weight varying between 400 and $500 \mathrm{~g}$, harvest should be performed when diameter fluctuates between 8.7 and $9.0 \mathrm{~cm}$, or when length fluctuates between 10.7 and $11.2 \mathrm{~cm}$. In the case of Keith, to obtain fruits with a harvest weight between 600 and $750 \mathrm{~g}$, harvest must be done when average diameter varies between 9.4 and $9.8 \mathrm{~cm}$ or when length is between 13.5 and $14.0 \mathrm{~cm}$, according to regression analysis.

la extracción total del árbol (Stassen et al. 1997, Silva et al. 2002).

Se han encontrado marcadas diferencias en la absorción de nutrimentos en los frutos entre los distintos cultivares e inclusive dentro de un mismo cultivar, lo cual es asociado a los distintos contenidos de nutrimentos que se presentan en cada sitio donde se desarrollaron los estudios (Catchpoole y Bally 1995, citados por Silva et al. 2002, Stassen et al. 1997, Silva et al. 2002). Los autores mencionados concuerdan en que la absorción es en gran medida dependiente de la edad del árbol. También, los requerimientos nutricionales de una plantación están relacionados con términos ampliamente utilizados en el campo de la fisiología vegetal, tales como grados día o grados temperatura, ya que es conocido que el desarrollo de los diferentes órganos y su demanda nutrimental es dependiente de la temperatura y el fotoperíodo (Misle 2003); estos factores concuerdan con lo mencionado por Sancho (1999), quien dice que la extracción de nutrimentos está ligada a factores internos y externos a la plantación.

En las investigaciones realizadas por Stassen et al. (1997), no solamente se detalla la absorción de los nutrimentos, sino que se muestra su distribución en las diferentes partes del árbol, sin embargo esta información ha sido generada en zonas de clima templado, donde el crecimiento del árbol se encuentra limitado por las 
condiciones climáticas, por lo que dicha absorción y distribución en las diferentes épocas del año podrían no coincidir con el comportamiento presentado en el trópico.

Las curvas de absorción de nutrimentos son una herramienta que permite conocer las necesidades o los momentos de máxima absorción y así ajustar los diferentes programas de fertilización, ya que mediante su expresión gráfica es más fácil determinar dichas necesidades (Bertsch 1995, Sancho 1999). Al ajustar la fertilización a la demanda se asegura una mayor calidad, especialmente en el caso del mango, ya que se sabe que en este tipo de fruto existe una estrecha relación entre el contenido de los diferentes nutrimentos y la calidad del fruto a exportar (Oosthuyse 1997, Donald et al. 2002).

El objetivo de este trabajo fue determinar, bajo condiciones tropicales, la fenología del desarrollo del fruto de 2 cultivares de mango: Tommy Atkins y Keith, ampliamente producidos en Costa Rica para exportación, las curvas de absorción de nutrimentos en dichos frutos y la correlación entre las diferentes variables fenológicas para obtener índicadores de manejo de utilidad práctica.

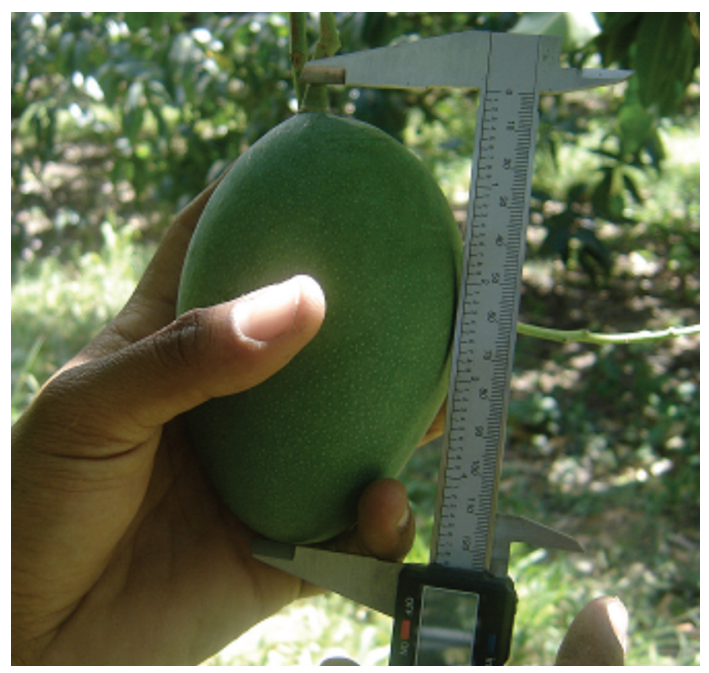

\section{MATERIALES Y MÉTODOS}

En 6 árboles de un mismo bloque, en la finca Manga Rica, Liberia, Guanacaste, Costa Rica, fueron colectados 4 frutos por árbol de los cultivares Tommy Atkins y Keith en 5 diferentes épocas de muestreo distribuido en 47, 61, 79, 93 y 110 días a partir del momento de máxima floración (DDF) para el cultivar Tommy Atkins y en 6 épocas para el cultivar Keith distribuido en 47, 61, 79, 93, 110 y 129 DDF, durante el ciclo de producción 2008-2009.

A cada fruto se le midieron las dimensiones de ancho y largo para cada época, con un calibrador milimétrico "vernier". Para la medición del largo de fruto no se le retiró el pedúnculo que unía el fruto a la planta madre y se midió el diámetro ecuatorial tal y como se muestra en la Figura 1.

Los frutos fueron pesados en fresco y se secaron en una estufa a $80^{\circ} \mathrm{C}$ por 3 días para extraer la humedad y luego obtener el peso seco.

Después de esto se unieron los frutos de cada parcela, se homogenizó la muestra y se les realizó el análisis de concentración de nutrimentos en el Laboratorio de Suelos y Foliares del Centro de Investigaciones Agronómicas (CIAUCR).

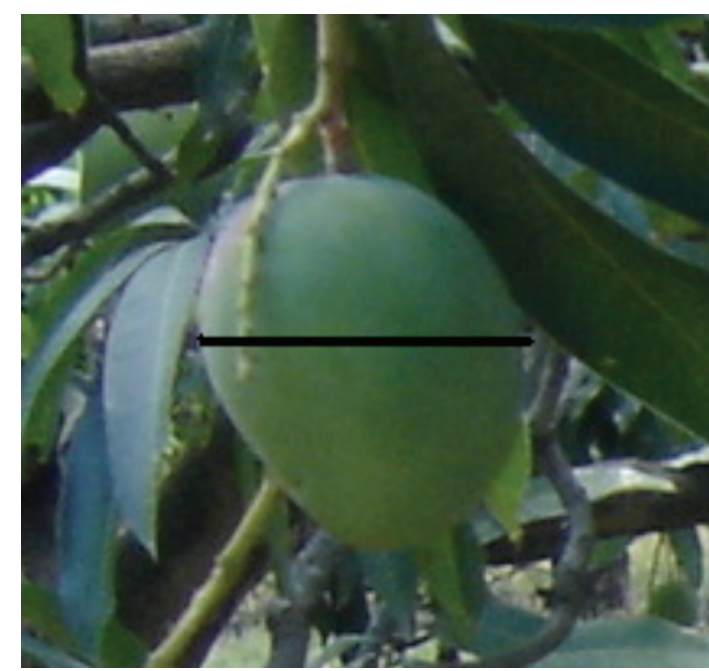

Fig. 1. Metodología de medición del largo y diámetro ecuatorial del fruto de mango. 
Una vez obtenidos los datos se relacionó el peso promedio de cada edad con su respectiva concentración de nutrimentos para conocer la absorción por kilogramo de fruta.

Con los datos obtenidos se realizaron las curvas de absorción de nutrimentos para cada variedad, asimismo se realizó una descripción del ciclo de crecimiento del fruto.

También se establecieron las correlaciones existentes entre las diferentes variables con el fin de determinar la factibilidad de usar las más sencillas como métodos de campo para ajustar fertilizaciones al relacionarlas con la absorción del fruto o como herramientas útiles para determinar la época de cosecha.

Cabe anotar que la finca realiza rutinariamente un monitoreo del estado nutricional, mediante análisis de tejido foliar a cada bloque y además utilizó riego ya que el ensayo se realizó en la época seca, por lo que se puede asegurar que a lo largo del ensayo, se contó con una plantación en condiciones óptimas.

\section{RESULTADOS Y DISCUSIÓN}

\section{Desarrollo del fruto}

Tommy Atkins: El fruto de mango cv. Tommy Atkins en sus primeros estados de desarrollo presenta un gran incremento en su tamaño específicamente el diámetro y largo, no así en su peso.

La época de mayor incremento de tamaño se da entre los 61 y 79 días después de floración (DDF), con un aumento de un 33\% del total del diámetro alcanzado a cosecha y un $29 \%$ en el largo del fruto, lo que refleja que antes de los 79 DDF el fruto ha alcanzado casi un $70 \%$ de su tamaño máximo promedio en lo que corresponde a la variable largo de fruto y un $74 \%$ correspondiente al diámetro (Figura 2).

El fruto tiende a expandirse a un ritmo más lento conforme se acerca a la época de cosecha, lo cual es aún más notorio en el último período evaluado, con un aumento de tan solo $7 \%$ de su diámetro y un $9 \%$ de su largo, aproximadamente.
Contrario al incremento en tamaño, el fruto en sus primeros estados de desarrollo aumenta de peso a un ritmo lento; al cabo de las primeras 2 etapas evaluadas el fruto tan solo ha acumulado cerca de un $6 \%$ del peso fresco total y cerca de un $4 \%$ de su peso seco.

En el período entre los 61 y 79 DDF el fruto comienza a presentar una tendencia de incremento en peso, no obstante, hasta antes de los 79 DDF el fruto tan solo ha acumulado un $31 \%$ de su peso fresco total y un $17 \%$ de su peso seco total, lo cual es contrastante con el incremento en las dimensiones alcanzadas a la misma fecha mencionadas anteriormente, esto es, $70 \%$ del largo y $74 \%$ del diámetro, lo que permite establecer que el fruto presenta 2 fases en su ciclo de desarrollo: la primera corresponde a expansión y la segunda al llenado de ese tejido formado.

El período que abarca de los 79 a $93 \mathrm{DDF}$ se muestra como el de mayor incremento en peso fresco y seco, 42 y $41 \%$, respectivamente, y de los 93 a los 110 DDF se mantiene el aumento en el peso seco (40\%), pero el peso fresco baja (27\%), lo cual refleja una disminución en el contenido de agua en las etapas cercanas a la cosecha. Cuando se suman los 2 últimos períodos evaluados el fruto de Tommy Atkins incrementa en ellos cerca de un $83 \%$ de su peso seco total y un $69 \%$ de su peso fresco con un leve aumento en sus dimensiones.

Keith: Al igual que los frutos de Tommy Atkins, los del cultivar Keith presentan 2 fases en su ciclo de desarrollo: una correspondiente a un incremento de su tamaño (expansión), la cual se localiza en etapas iniciales del ciclo de desarrollo y la otra correspondiente a un incremento de peso que constituiría la fase de "llenado", que ocurre en etapas cercanas a la cosecha.

La diferencia que presenta este cultivar es que los cambios de tendencia tanto en tamaño como en peso se dan un poco más tarde, a los 93 DDF (Figura 2).

Al tener un ciclo de desarrollo más largo, el cultivar Keith alcanza mayores dimensiones y peso. 


\section{Tommy Atkins}
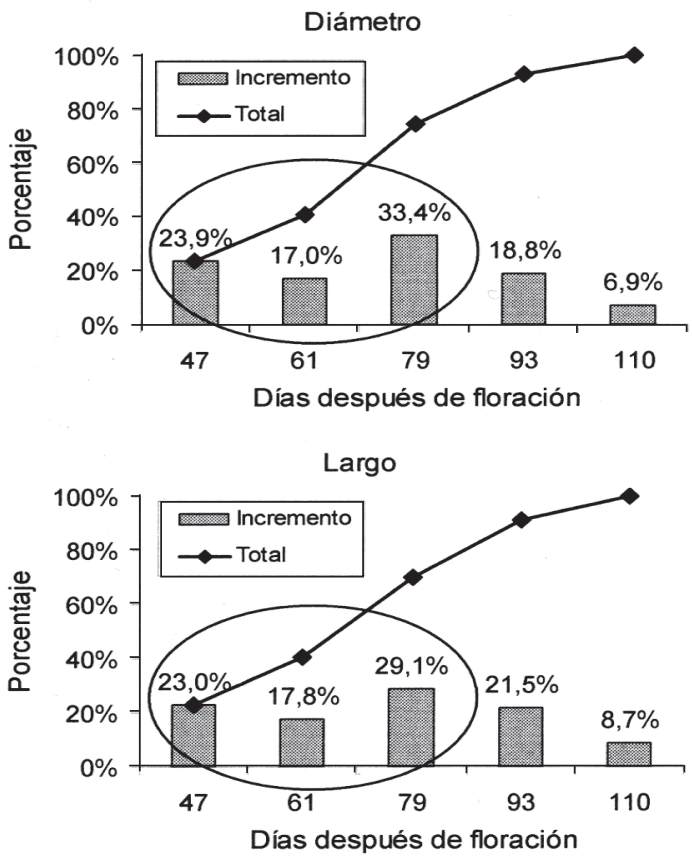

Peso seco

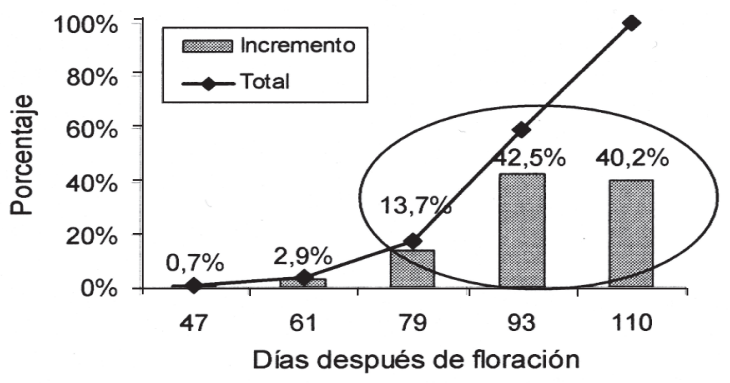

Peso fresco

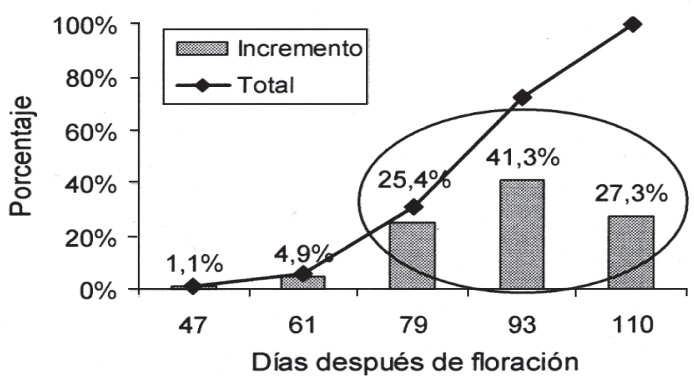

Keith
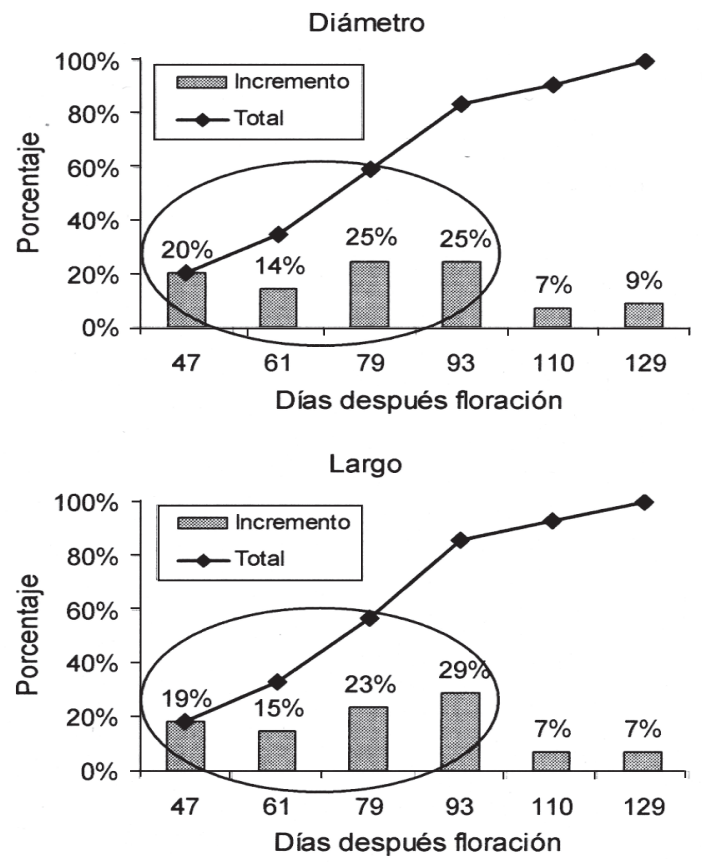

Peso seco

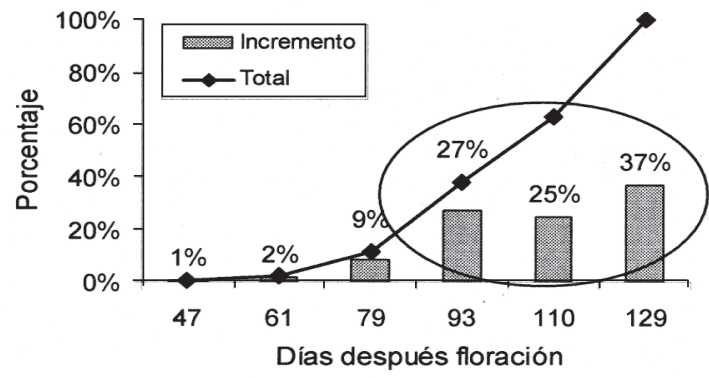

Peso fresco

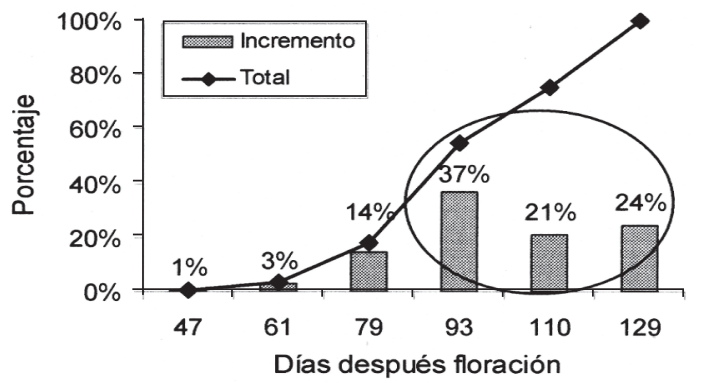

Fig. 2. Desarrollo del fruto de mango (Mangifera indica) de 2 cultivares, Tommy Atkins y Keith, según el incremento porcentual total y por época de muestreo del diámetro, largo y peso. 
De estos resultados se desprende la importancia de suministrar adecuadamente en fases tempranas del ciclo de desarrollo los nutrientes, el agua y cualquier otro factor que limite el incremento en tamaño del fruto; la carencia de alguno de estos factores posiblemente resulte en fruta pequeña y también el realizar la cosecha antes de tiempo puede resultar en un menor rendimiento de la producción, debido a que el incremento en peso se da en las etapas finales.

\section{Absorción de nutrimentos}

\section{$N, P$ y K}

Para ambos cultivares, en las primeras etapas el fruto absorbe pequeñas cantidades de $\mathrm{N}$, P y K y el incremento en la absorción de estos nutrimentos se inicia a partir del período entre los 61 y 79 DDF; para esta fase el fruto absorbe más del doble de las cantidades absorbidas en las 2 evaluaciones anteriores (Figura 3 ).

La absorción de N, P y K por parte del cultivar Keith, se diferencia de la presentada por el cultivar Tommy Atkins, en que Keith tiene un ciclo de desarrollo del fruto más largo, por lo tanto distribuye en los últimos 3 períodos la absorción equivalente a la presentada en los 2 últimos por Tommy Atkins.

Por este motivo se sugiere que las aplicaciones de estos 3 elementos en etapa de fructificación se realicen en el período posterior a los 79 DDF en el caso de fertilizaciones foliares y anterior a los 61 DDF en el caso de fertilizaciones al suelo, ya que el árbol tardaría un poco en trasladar dichos nutrimentos hasta los frutos. No obstante, es importante tener cuidado con las aplicaciones de $\mathrm{N}$ en etapa de fructificación, ya que se ha reportado que altas concentraciones de este elemento pueden afectar la calidad de fruta (Avilán 2008). Se debe destacar que la absorción presentada en este estudio es la referente al fruto y no la del árbol, por lo que este estudio es una referencia de fertilización con respecto a las necesidades del fruto y no de la plantación total.
La absorción de estos 3 nutrimentos $(\mathrm{N}$, $\mathrm{P}$ y K) contrasta con lo encontrado en el incremento de tamaño (diámetro y largo) del fruto, pero corresponde con la tendencia presentada en cuanto al peso.

La tendencia de absorción de nutrimentos refleja el impacto que podría tener una deficiencia nutrimental sobre un factor de calidad del fruto tan importante como el peso alcanzado a la cosecha, por lo que es relevante suplementar los nutrimentos en las cantidades requeridas y en el momento adecuado.

\section{Ca y $M g$}

Para los 2 cultivares la absorción de $\mathrm{Ca}$ y $\mathrm{Mg}$ presenta un patrón de absorción similar al encontrado para N, P y K, lo cual indica que las épocas de menor absorción son las iniciales y las de mayor absorción se encuentran al final del ciclo de desarrollo del fruto (Figura 3).

Para el cultivar Tommy Atkins un 77\% del total de $\mathrm{Ca}$ es absorbido en las últimas 2 etapas (93 y $110 \mathrm{DDF}$ ) y un $80 \%$ para el caso del Mg; la última etapa es la que presenta una mayor absorción de ambos elementos (Figura 3). Por otro lado, el cultivar Keith, parece que adelanta la absorción de Ca, ya que a los 61 DDF ha absorbido el doble o más del doble del equivalente porcentual con respecto a los otros elementos con patrón de absorción similar (Figura 3) además, presenta una importante absorción a los 79 DDF (19\%), pero muestra una disminución a los 110 DDF, lo cual no sucede para el cultivar Tommy Atkins.

\section{Elementos menores y azufre}

Algunos de estos elementos muestran un patrón de absorción similar al presentado para elementos mayores, es decir su mayor absorción se localiza en etapas cercanas a la cosecha, pero otros elementos se salen de este patrón y muestran un comportamiento particular.

\section{S, Си у $\mathrm{B}$}


Tommy Atkins

N

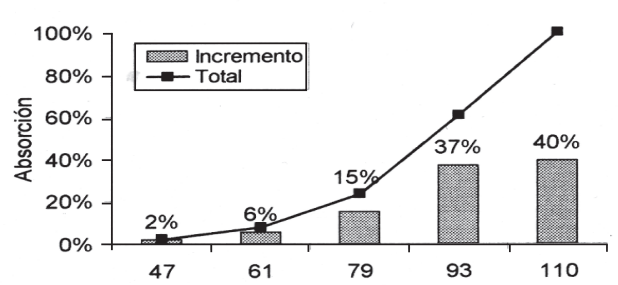

$P$

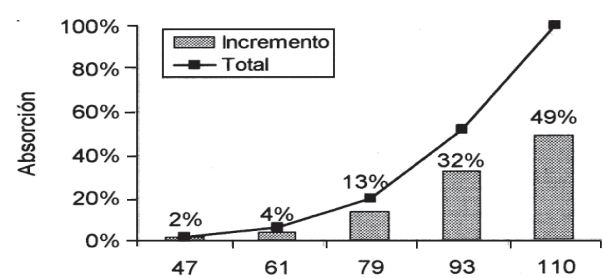

K

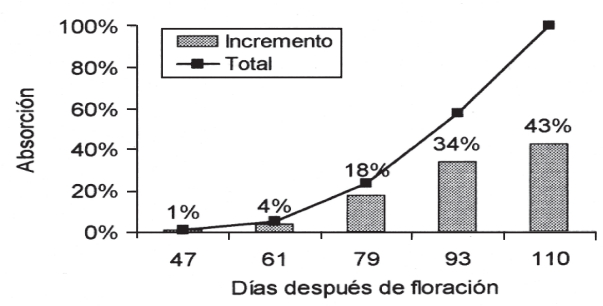

$\mathrm{Ca}$
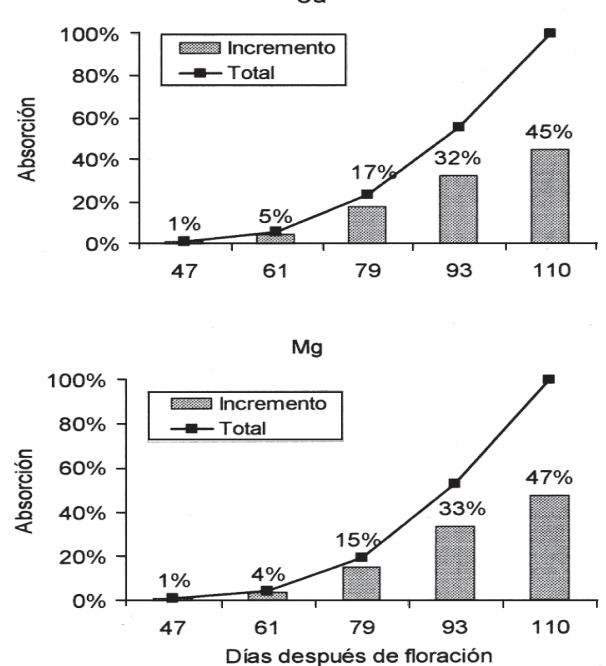

Keith

N

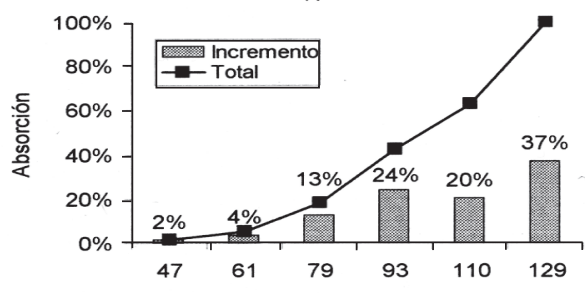

P

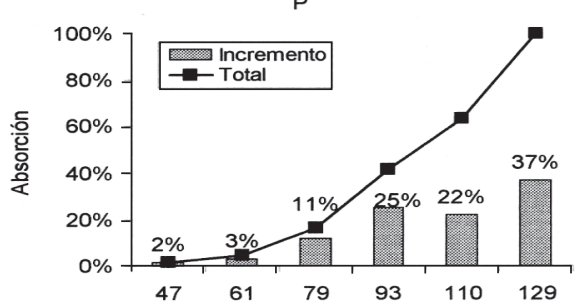

K
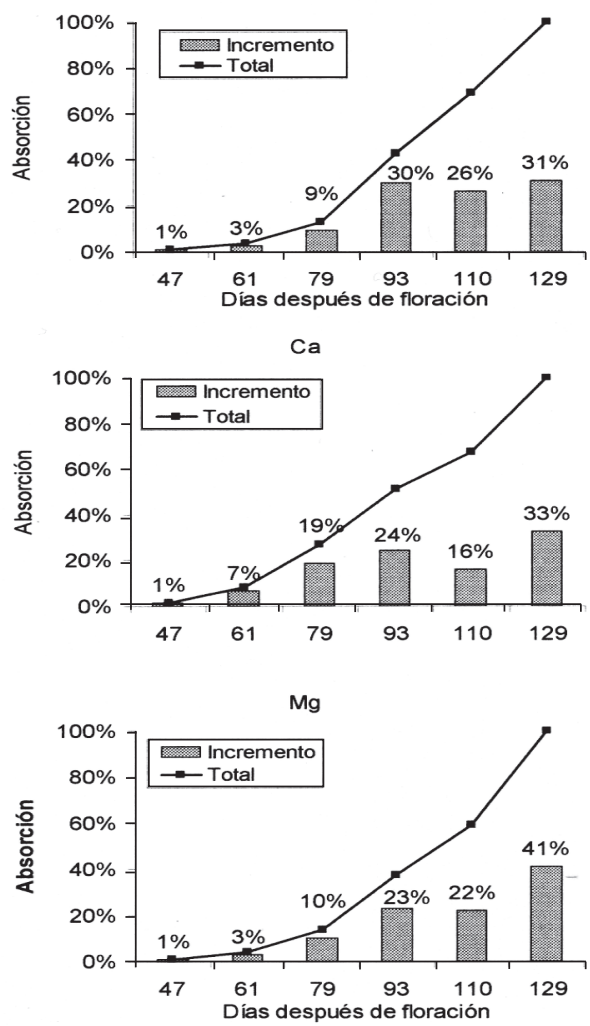

Fig. 3. Curvas de absorción porcentual de N, P, K, Ca y Mg del fruto de mango cvs. Tommy Atkins y Keith durante su ciclo de desarrollo total y por época de muestreo. 
Tommy Atkins: La absorción de estos 3 elementos al inicio del ciclo de desarrollo no se diferencia del patrón observado para macronutrimentos (47 y $61 \mathrm{DDF}$ ), sin embargo a los 79 DDF el fruto absorbe de cada nutrimento una cantidad mayor (24\% del total) que cualquiera de los otros y en el período entre los 79 y 93 DDF se da la máxima absorción de $\mathrm{S}, \mathrm{Cu}$ y $\mathrm{B}$, (54\%, 64\% y 52\% del total, respectivamente) (Figura 4). Sin embargo, su principal diferencia con respecto a elementos mayores radica en que en la última etapa el fruto disminuye su demanda hasta valores de un $13 \%$ para $\mathrm{S}, 5 \%$ para $\mathrm{Cu}$ y $15 \%$ para el caso del B.

Keith: La absorción de $\mathrm{S}$ y $\mathrm{Cu}$ difiere de lo presentado por Tommy Atkins en que Keith distribuye más su demanda o absorción en los últimos 3 períodos.

El elemento que se comporta de una manera particular en el cultivar Keith es el B, ya que la etapa de mayor absorción fue la última, con una absorción final de más de un $70 \%$ del total requerido durante el ciclo de desarrollo del fruto.

$\mathrm{Al}$ parecer en los 2 cultivares evaluados la absorción de B se concentra en una etapa específica de su ciclo de desarrollo, para el caso de Keith en la última etapa como ya se mencionó y para el caso de Tommy Atkins a los 93 DDF (Figura 4).

En el caso de Rossetto et al. (2001), citados por Raja (2007) encontraron que un árbol de Tommy Atkins con una buena nutrición de este elemento puede producir hasta el doble de un árbol con deficiencia, lo cual afirma la importancia de conocer las necesidades y las épocas en las que es requerido.

Por otro lado en el cultivar Tommy Atkins las aplicaciones de $\mathrm{S}, \mathrm{Cu}$ y $\mathrm{B}$ deben suministrarse antes del período de los 61 a 79 DDF y extenderse como máximo hasta los 93 DDF, aplicaciones al final del ciclo podrían no resultar efectivas, ya que en la última etapa el fruto disminuye su absorción de estos 3 elementos.

\section{Fe y Mn}

Tommy Atkins: La absorción de estos 2 nutrimentos por parte de Tommy Atkins se da principalmente al final del ciclo de desarrollo del fruto, con un fuerte acúmulo de $85 \%$ del Fe y $76 \%$ del Mn en la última etapa (Figura 5). Es por esto que podría considerarse que las aplicaciones foliares de estos elementos deberían realizarse en etapas tardías del ciclo de desarrollo.

Keith: En el caso del cultivar Keith la absorción de Fe y Mn también se da hacia el final del ciclo, pero contrario a Tommy Atkins no se concentra en un solo período y aparenta absorber de manera antagónica ambos elementos, ya que a una mayor absorción de Fe corresponde una baja absorción de Mn y viceversa; debido a esto es recomendable no realizar aplicaciones simultáneas de estos 2 elementos.

\section{Zn}

Tommy Atkins: La absorción de Zn se caracteriza por concentrarse en el período central del ciclo en la etapa que van de los 61 a los 79 DDF con más de un $80 \%$ del total requerido (Figura 5).

Keith: Este cultivar se diferencia de Tommy Atkins en que no concentra la absorción de Zn en una sola etapa, sino que la distribuye a lo largo de su ciclo de desarrollo, con una baja absorción (5\%) hasta los 61 DDF (Figura 5).

De acuerdo al patrón mostrado en la curva de absorción en ambos cultivares las aplicaciones de $\mathrm{Zn}$ deberían realizarse antes del período comprendido entre 61 y 79 DDF, además las aplicaciones tardías de este elemento en el cultivar Tommy Atkins podrían no ser efectivas, porque en las últimas etapas absorbe pequeñas cantidades del elemento.

Tal y como se mencionó anteriormente las curvas de absorción basadas en la edad cronológica, tienen el inconveniente que no toman en cuenta las diferentes condiciones que pueden alterar el desarrollo del cultivo (Misle 2003). 
Tommy Atkins

S

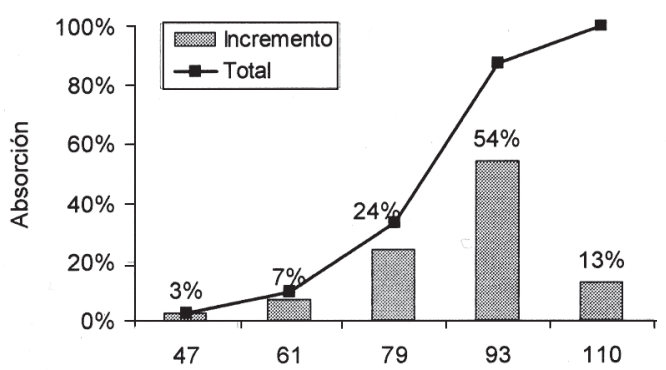

$\mathrm{Cu}$

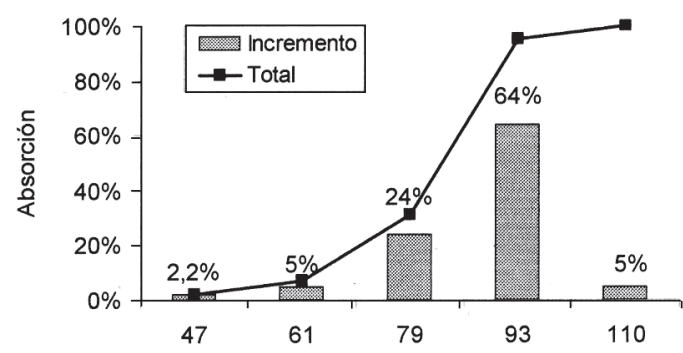

B

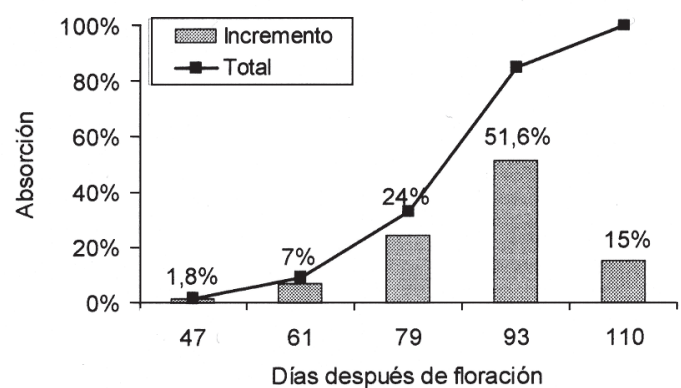

Keith

S

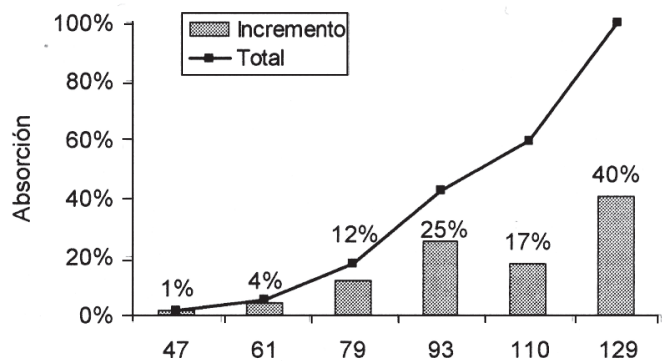

$\mathrm{Cu}$

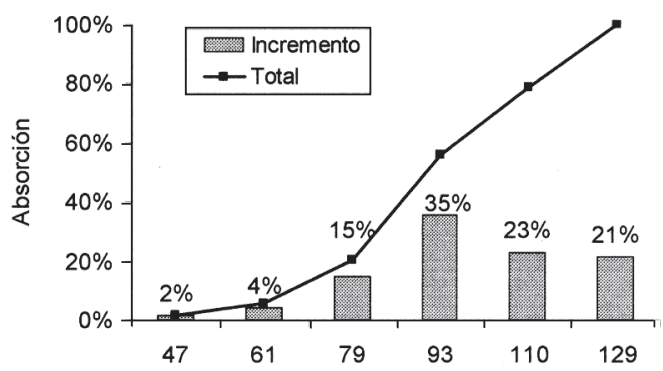

B

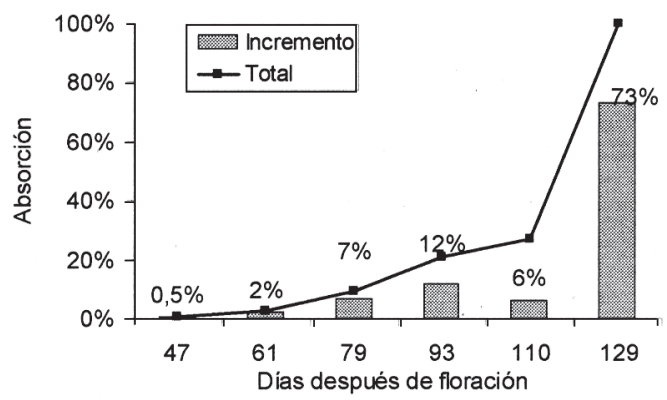

Fig. 4. Curvas de absorción porcentual de S, Cu y B durante el ciclo de desarrollo del fruto de mango y su respectivo incremento en cada etapa (cvs. Tommy Atkins y Keith). 
Tommy Atkins

$\mathrm{Fe}$

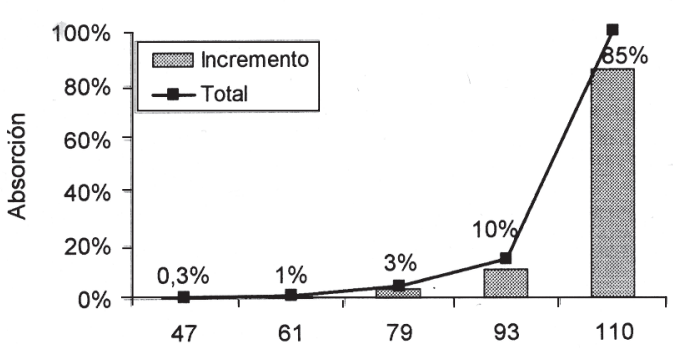

$\mathrm{Mn}$

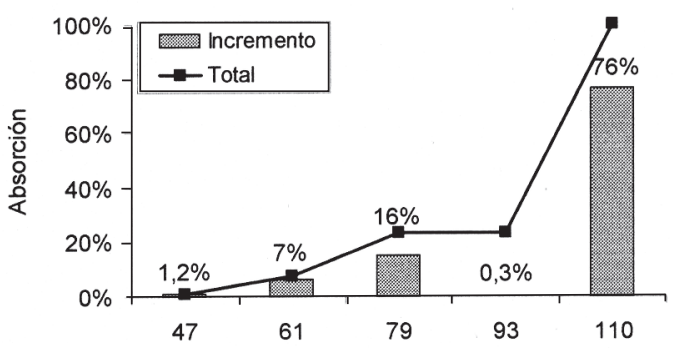

Zn

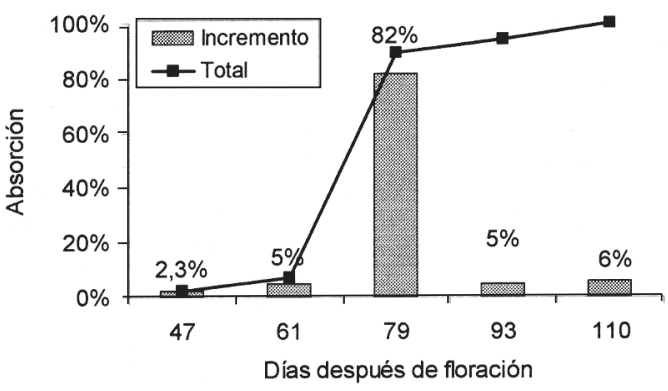

Keith

$\mathrm{Fe}$

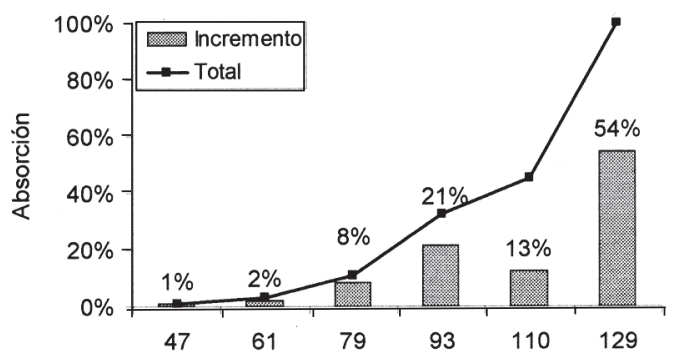

$\mathrm{Mn}$

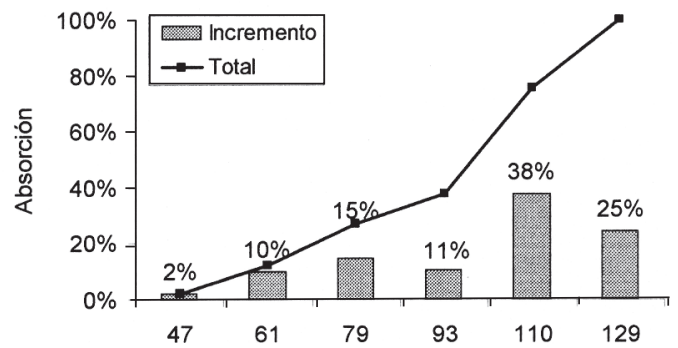

Zn

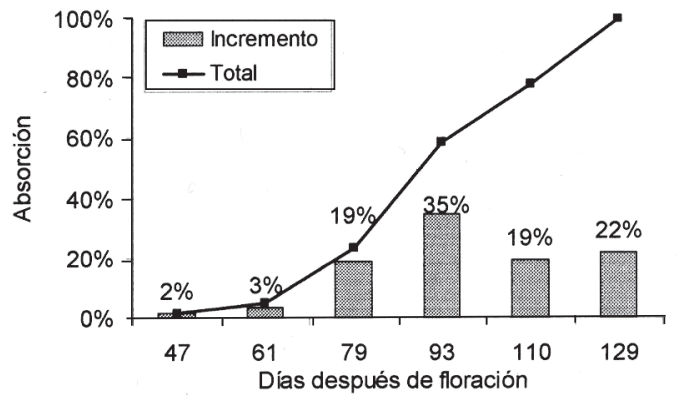

Fig. 5. Curvas de absorción porcentual de Fe, Mn y Zn en cada etapa del ciclo de desarrollo del fruto de mango cvs. Tomy Atkins y Keith. 
Con el objetivo de disminuir el error al adaptar las curvas de absorción a otras plantaciones o a otras condiciones climáticas, (Figura 6) se muestran los valores promedio del diámetro y largo de fruto correspondientes con cada una de las etapas, para que con estos resultados y mediciones de estas variables a nivel de campo, se pueda estimar el estado de la curva de absorción en que se encuentra una determinada plantación

\section{Tommy Atkins}
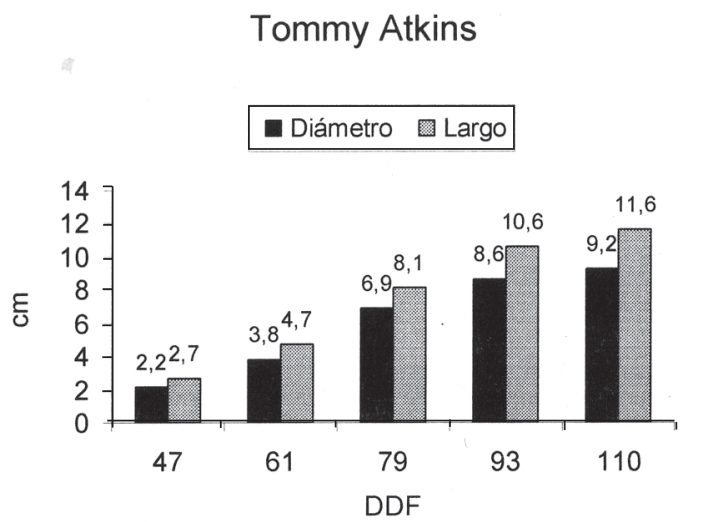

y así se realicen las correcciones pertinentes al estado nutricional y requerimientos.

Además, al integrar los factores climáticos de mayor relevancia durante el período del ensayo, en los Cuadros 1 y 2 se muestran los grados día temperatura que, según estimaciones hechas a partir de la fórmula de Ometo (Yzarra et al. 2009), se requirieron en cada etapa de desarrollo del fruto.

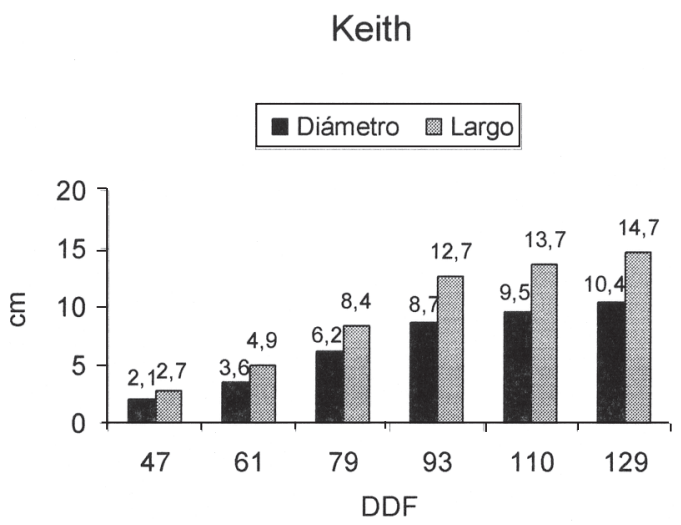

Fig. 6. Valores promedio de diámetro y largo obtenidos en cada etapa de muestreo durante la realización del ensayo.

Cuadro 1. Cantidad de nutrimentos absorbida por kilogramo de fruta fresca en cada etapa de muestreo y en el total del ciclo de desarrollo para el cultivar Tommy Atkins en la Finca Manga Rica, Liberia, Guanacaste, Costa Rica. Período 2008-2009.

\begin{tabular}{|c|c|c|c|c|c|c|c|c|c|c|c|c|}
\hline \multirow[b]{3}{*}{ DDF } & \multirow[b]{3}{*}{ Grados día } & \multirow[b]{3}{*}{$\mathrm{N}$} & \multicolumn{9}{|c|}{ Cantidad absorbida } & \multirow[b]{3}{*}{ B } \\
\hline & & & \multicolumn{5}{|c|}{ mg.kg ${ }^{-1}$ de fruta fresca } & \multicolumn{4}{|c|}{ ug. $\mathrm{kg}^{-1}$ de fruta fresca } & \\
\hline & & & $\mathrm{P}$ & $\mathrm{K}$ & $\mathrm{Ca}$ & $\mathrm{Mg}$ & $S$ & $\mathrm{Fe}$ & $\mathrm{Cu}$ & $\mathrm{Zn}$ & $\mathrm{Mn}$ & \\
\hline $0-38^{*}$ & 559 & & & & & & & & & & & \\
\hline 47 & 132 & 24,7 & 3,5 & 20,0 & 3,2 & 1,8 & 1,9 & 25,1 & 24,3 & 27,0 & 41,4 & 12,3 \\
\hline 61 & 203 & 85,3 & 11,6 & 83,0 & 16,3 & 8,7 & 7,0 & 86,7 & 78,5 & 86,7 & 268,4 & 61,9 \\
\hline 79 & 272 & 249,3 & 38,3 & 360,1 & 66,7 & 37,3 & 24,5 & 363,1 & 343,5 & 1059,8 & 804,7 & 225,7 \\
\hline 93 & 230 & 644,4 & 101,7 & 885,2 & 159,4 & 101,7 & 64,4 & 1254,8 & 1051,4 & 1119,2 & 814,0 & 576,5 \\
\hline 110 & 215 & 1065,3 & 198,3 & 1541,3 & 289,0 & 192,7 & 73,7 & 8500,0 & 1105,0 & 1190,0 & 3456,7 & 680,0 \\
\hline Total & 1611 & 1065,3 & 198,3 & 1541,3 & 289,0 & 192,7 & 73,7 & 8500,0 & 1105,0 & 1190,0 & 3456,7 & 680,0 \\
\hline
\end{tabular}

Los grados día correspondientes a esta etapa fueron estimados a través del promedio, por no disponer de la información meteorológica directa que se empezó a registrar al inicio de los muestreos. El cálculo de los grados día se hizo según la fórmula de Ometo (citado por Yzarra et al. 2009). 
Cuadro 2. Cantidad de nutrimentos absorbida por kilogramo de fruta fresca en cada etapa de muestreo y en el total del ciclo de desarrollo para el cultivar Keith en la Finca Manga Rica, Liberia, Guanacaste, Costa Rica. Período 2008-2009.

\begin{tabular}{|c|c|c|c|c|c|c|c|c|c|c|c|c|}
\hline \multirow[b]{3}{*}{ DDF } & \multirow[b]{3}{*}{ Grados día } & \multirow[b]{3}{*}{$\mathrm{N}$} & \multicolumn{9}{|c|}{ Cantidad absorbida } & \multirow[b]{3}{*}{ B } \\
\hline & & & \multicolumn{4}{|c|}{ mg.kg ${ }^{-1}$ de fruta fresca } & \multirow[b]{2}{*}{$\mathrm{S}$} & \multicolumn{4}{|c|}{ ug. $\mathrm{kg}^{-1}$ de fruta fresca } & \\
\hline & & & $\mathrm{P}$ & $\mathrm{K}$ & $\mathrm{Ca}$ & $\mathrm{Mg}$ & & $\mathrm{Fe}$ & $\mathrm{Cu}$ & $\mathrm{Zn}$ & $\mathrm{Mn}$ & \\
\hline $0-38^{*}$ & 559 & & & & & & & & & & & \\
\hline 47 & 132 & 17,5 & 2,0 & 14,3 & 2,0 & 1,5 & 1,2 & 20,9 & 16,2 & 16,0 & 39,8 & 7,6 \\
\hline 61 & 203 & 56,8 & 6,0 & 58,8 & 11,6 & 6,0 & 4,5 & 75,3 & 51,4 & 47,8 & 255,7 & 41,8 \\
\hline 79 & 272 & 192,0 & 20,3 & 207,6 & 37,5 & 21,3 & 15,1 & 281,0 & 187,3 & 228,9 & 572,3 & 150,9 \\
\hline 93 & 230 & 450,9 & 51,5 & 679,9 & 71,0 & 56,8 & 37,3 & 816,6 & 514,8 & 568,0 & 798,8 & 337,3 \\
\hline 110 & 215 & 665,9 & 78,5 & 1096,3 & 93,1 & 90,1 & 52,3 & 1134,1 & 727,0 & 756,1 & 1599,4 & 436,2 \\
\hline 129 & 275 & 1061,2 & 124,6 & 1587,1 & 138,4 & 152,3 & 87,7 & 2491,4 & 922,7 & 968,9 & 2122,3 & 1614,8 \\
\hline Total & 1327 & 1061,2 & 124,6 & 1587,1 & 138,4 & 152,3 & 87,7 & 2491,4 & 922,7 & 968,9 & 2122,3 & 1614,8 \\
\hline
\end{tabular}

Los grados día correspondientes a esta etapa fueron estimados a través del promedio, por no disponer de la información meteorológica directa que se empezó a registrar al inicio de los muestreos. El cálculo de los grados día se hizo según la fórmula de Ometo (citado por Yzarra et al. 2009).

\section{Absorción total}

En los Cuadros 1 y 2, se detalla la absorción promedio presentada en cada etapa evaluada y la absorción total del ciclo de desarrollo del fruto para los diferentes elementos en los 2 cultivares.

Como se puede observar, el elemento absorbido en mayor cantidad por el fruto de mango es el $\mathrm{K}$ y luego el N, lo cual concuerda con lo encontrado por Gontijo (1982), citado por Vega y Molina (1999), además se encuentran en una relación $\mathrm{N} / \mathrm{K}$ de aproximadamente $1 / 1,5$ respectivamente.

El Ca, P y Mg son los siguientes elementos absorbidos según cantidad (valores entre 0,14 y $0,29 \mathrm{~g} \cdot \mathrm{kg}^{-1}$ ) sin embargo su secuencia varía según el cultivar: ese orden $\mathrm{Ca}, \mathrm{P}$ y $\mathrm{Mg}$ ocurre para Tommy Atkins mientras que para Keith la secuencia es Mg, Ca y P. En general, el fruto de Keith absorbe menores cantidades que Tommy Atkins durante el total de su ciclo de desarrollo, a pesar de que alcanza mayores dimensiones.

El S es otro elemento que debe ser considerado en la fertilización de mango de ambos cultivares, ya que el fruto demanda cantidades importantes de este elemento (0,07-0,09 g.kg-1 de fruta fresca).

También se puede apreciar que los microelementos absorbidos en mayor cantidad por el fruto de mango en ambos cultivares son $\mathrm{Fe}$ y $\mathrm{Mn}$. De los 3 restantes, Zn, Cu y B resalta una mayor demanda de $\mathrm{B}$ en el cultivar Keith con respecto a Tommy Atkins (Cuadros 1 y 2).

La demanda de $\mathrm{N}$ y K en ambos cultivares es similar en términos de $\mathrm{g}$ del elemento absorbidos por kg de fruta fresca, sin embargo Tommy Atkins demanda una mayor cantidad de algunos elementos como el $\mathrm{P}, \mathrm{Ca}, \mathrm{Mg}, \mathrm{Fe}$; por lo que se debe diferenciar entre cada cultivar a la hora de tomar criterios de fertilización.

Debido a que las condiciones climáticas afectan el desarrollo del fruto y por ende la absorción de nutrimentos, mediante el uso de los grados día temperatura sería posible transferir la información de las curvas de absorción a diferentes localidades o ciclos de producción. En los Cuadros 1 y 2 se detallan los grados día temperatura presentados durante la realización del ensayo. Dicha información también es útil para predecir la época de cosecha del fruto. 


\section{Correlación entre variables evaluadas}

Tal y como se muestra en la Figura 7, existe una gran afinidad o correlación entre las diferentes variables fenológicas evaluadas en los 2 cultivares en estudio.

Esto hace que las variables evaluadas se conviertan en indicadores confiables en la determinación del estado de desarrollo del fruto y la absorción de nutrimentos, de las cuales el diámetro ecuatorial y el largo del fruto tienen gran importancia práctica, ya que son mediciones fáciles de realizar en el campo.

En el caso de no contar con datos climáticos, estas variables pueden ser utilizadas en la determinación del estado de desarrollo del fruto y como herramienta para adaptar las curvas de absorción de nutrimentos a diferentes condiciones.

Existe un comportamiento lineal entre el peso fresco y el peso seco (Figura 7), con un coeficiente de correlación $\left(\mathrm{r}^{2}\right)$ de 0,94 para Tommy Atkins y 0,96 para Keith. Esta asociación sirve para poder relacionar el peso fresco con la absorción, ya que las curvas de absorción se obtienen de la concentración promedio de los elementos (análisis de laboratorio) y el peso seco promedio del fruto.

Para el caso de las variables de longitud (largo y ancho de fruto), se determinó que también pueden usarse para hacer un estimado de la absorción de nutrimentos por parte del fruto, debido a su relación con el peso seco; además para determinar la época de cosecha, ya que correlacionan muy bien con el peso fresco del fruto. Estas variables presentan la ventaja que el fruto no debe ser cosechado para realizar el muestreo.

Según los datos de correlación y regresión, para obtener frutos del cultivar Tommy Atkins con pesos frescos que varíen entre 400 y $500 \mathrm{~g}$ se debe realizar la cosecha cuando estos presenten un diámetro que fluctúe entre 8,7 y $9,0 \mathrm{~cm}$ respectivamente. La variable de largo de fruto también puede ser utilizada para estos fines. Para obtener frutos con los pesos mencionados (400 y $500 \mathrm{~g}$ ), éstos deben cosecharse cuando la fruta alcanza un largo entre $10,7 \mathrm{~cm}$ y $11,2 \mathrm{~cm}$ respectivamente (Figura 7).

Para el caso del cultivar Keith, para obtener frutos con un peso a la cosecha que fluctúe entre 600 y $750 \mathrm{~g}$ se debe cosechar la fruta cuando el diámetro varíe entre 9,4 y $9,8 \mathrm{~cm}$ de diámetro y/o entre 13,5 y 14,0 cm de largo, según los datos obtenidos de la ecuación obtenida en el análisis de correlación (Figura 7).

Es importante destacar que los valores $\left(\mathrm{R}^{2}\right)$ de los coeficientes de correlación encontrados en todos los casos fueron superiores a 0,95 , a excepción del peso fresco y peso seco en el cultivar Keith $(0,94)$, estos datos son altamente significativos ( $p<0,00001)$, lo cual respalda el uso de las variables evaluadas en la estimación del peso, la absorción y la época de cosecha (Figura 7). 


\section{Tommy Atkins}
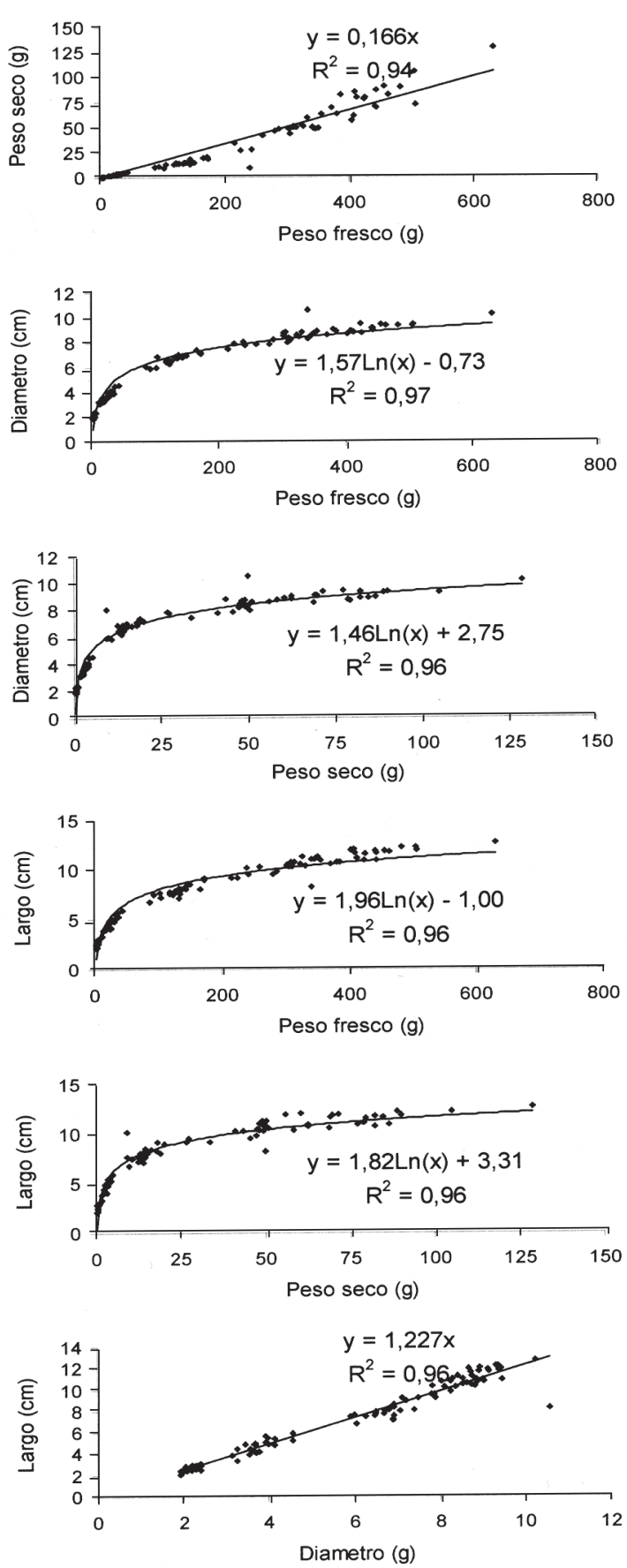

\section{Keith}
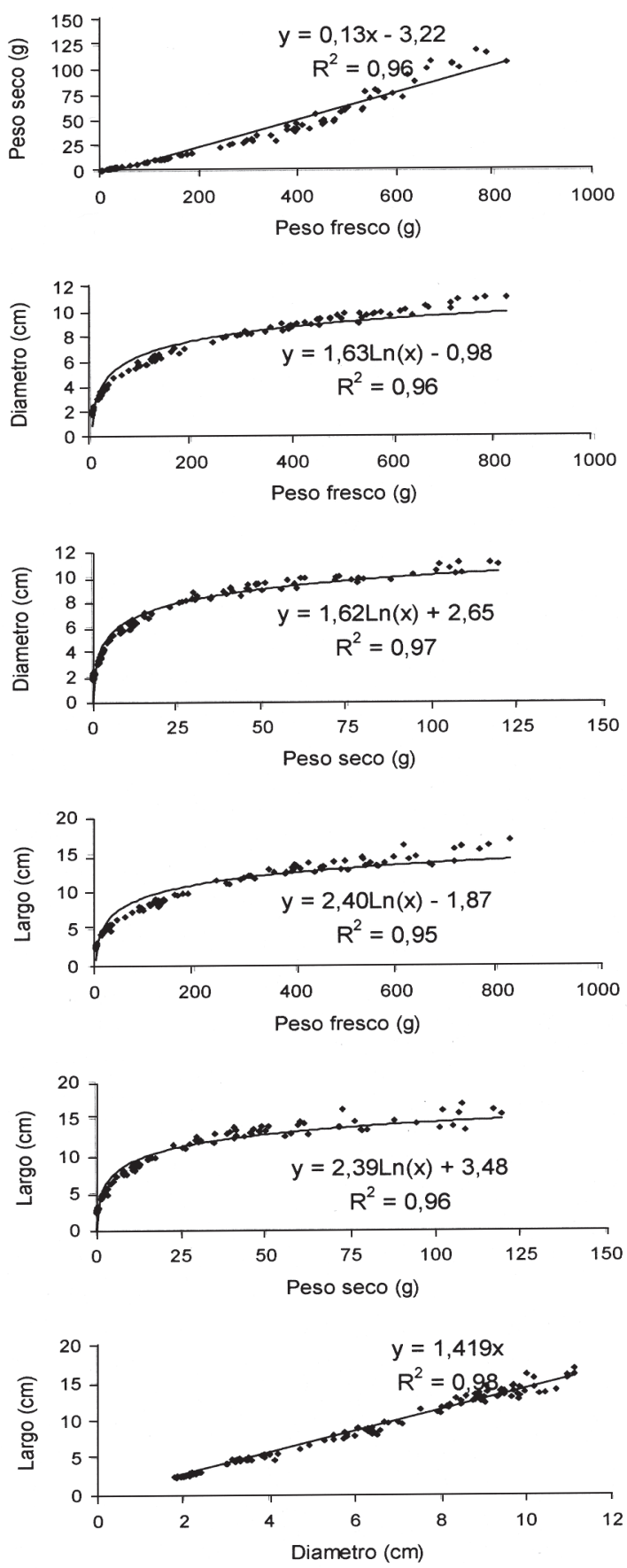

Fig. 7. Correlación y regresión entre las diferentes variables evaluadas en el fruto de mango cvs. Tommy Atkins y Keith, su respectiva ecuación y valor de $\mathrm{R}^{2}$. 


\section{LITERATURA CITADA}

AVILÁN L. 1999. Fertilización del mango en el trópico. Informaciones Agronómicas. Instituto de la Potasa y el Fósforo. Canadá p.1-10.

AVILÁN L. 2008. Nutrición y fertilización del mango. Internacional Plant Nutrition Institute. 73 p.

BERTSCH F. 1995. La fertilidad de los suelos y su manejo. San José, C.R. ACCS. 157 p.

DONALD J., RODRÍGUEZ M., SÁNCHEZ P., MORA A., CÁRDENAS E. 2002. Foliar fertilization of mango (cv Haden) for the control of malformation. Acta Horticulturae 594:667-673 p.

MISLE E. 2003. Caracterización termofisiológica del ritmo de absorción de nutrientes del melón (Cucumis melo L. var reticulatus Naud). Cien. Inv. Agr. 30(1):3950 .

OOSTHUYSE S. 1997. Relationship between leaf nutrient concentrations and cropping or fruit quality in mango. S.A. Mango Growers' Association Yearbook 17:1-15.

PONCHNER S., ROJAS R., BORNEMISZA E. 1993. Variación estacional de nutrimentos en árboles de mango (Mangifera indica) en tres suelos del pacífico seco de Costa Rica: I Macronutrimentos. Agronomía Costarricense 17(2):21-30.
RAJA E. 2007. Genotypic differences in tolerance to B deficiency in mango in India. Advances in Plant and Animal Boron Nutrition. 213-224 p.

RÍOS R., CORELLA F. 1999. Manejo de la nutrición y fertilización del mango en Costa Rica. XI Congreso Nacional Agronómico / III Congreso Nacional de Suelos.

SANCHO H. 1999. Curvas de absorción de nutrientes: importancia y uso en los programas de fertilización. Instituto de la Potasa y el Fósforo. Informaciones Agronómicas 36:11-13.

SILVA D., QUAGGIO J., DA COSTA P., DE QUEIROZ A., DE JESUS A. 2002. Nutrição e adubação In: A cultura da mangueira. Editores: de Carvalho, P y de Queiroz, A. EMBRAPA. 454 p.

STASSEN P., VANVUUREN J., DAVIE S. 1997. Macro elements in mango trees: uptake and distribution. S.A. Mango Growers' Association Yearbook 17:16$19 \mathrm{p}$.

VEGA E., MOLINA E. 1999. Fertilización nitrogenada en el cultivo de mango var. Tommy Atkins, en Guanacaste, Costa Rica. Agronomía Costarricense 23(1):37-44.

YZARRA W., TREBEJO I., NORIEGA V. 2009. Evaluación de unidades térmicas para el crecimiento y desarrollo del cultivo de maíz amarillo duro (Zea mays L.) en la costa central del Perú. Revista Peruana Geoatmosférica RPGA (1):1-10. 